DOI 10.4171/JEMS/371

Kim Dang Phung · Gengsheng Wang

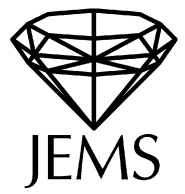

\title{
An observability estimate for parabolic equations from a measurable set in time and its applications
}

Received July 23, 2011 and in revised form November 5, 2011

\begin{abstract}
This paper presents a new observability estimate for parabolic equations in $\Omega \times(0, T)$, where $\Omega$ is a convex domain. The observation region is restricted over a product set of an open nonempty subset of $\Omega$ and a subset of positive measure in $(0, T)$. This estimate is derived with the aid of a quantitative unique continuation at one point in time. Applications to the bang-bang property for norm and time optimal control problems are provided.
\end{abstract}

Keywords. Parabolic equations, observability estimate, quantitative unique continuation, bangbang property

\section{Introduction and main result}

Let $\Omega$ be a bounded, convex and open subset of $\mathbb{R}^{n}, n \geq 1$, with boundary $\partial \Omega$. Let $T>0$. We consider the following parabolic equation:

$$
\begin{cases}\partial_{t} u-\Delta u+a u+b \cdot \nabla u=0 & \text { in } \Omega \times(0, T), \\ u=0 & \text { on } \partial \Omega \times(0, T), \\ u(\cdot, 0) \in L^{2}(\Omega) . & \end{cases}
$$

Here $b \in L^{\infty}(\Omega \times(0, T))^{n}, a \in L^{\infty}\left(0, T ; L^{q}(\Omega)\right)$ with $q \geq 2$ for $n=1$, and $q>n$ for $n \geq 2$. Clearly, it defines a well-posed problem in the sense of Hadamard, that is,

- for any $u_{0} \in L^{2}(\Omega)$, there is a unique solution $u \in C\left([0, T] ; L^{2}(\Omega)\right)$ of (1.1) with $u(\cdot, 0)=u_{0}$;

- the solution $u$ depends continuously on the initial value.

The above continuous dependence and uniqueness can be derived from the estimate

$$
\left.\int_{\Omega}|u(x, t)|^{2} d x \leq e^{C_{0} t\left(\|a\|_{L^{\infty}\left(0, T ; L^{q}(\Omega)\right)}^{2}+\|b\|_{L^{\infty}(\Omega \times(0, T))}^{2}\right.}\right) \int_{\Omega}|u(x, 0)|^{2} d x \quad \forall t \in[0, T],
$$

where $C_{0}$ is a positive constant depending only on $\Omega, n$ and $q$.

K. D. Phung: Université d'Orléans, Laboratoire MAPMO, CNRS UMR 7349, Fédération DenisPoisson, FR CNRS 2964, Bâtiment de mathématiques, B.P. 6759, 45067 Orléans Cedex 2, France; e-mail: kim_dang_phung@yahoo.fr

G. Wang: School of Mathematics and Statistics, Wuhan University, Wuhan 430072, China; e-mail: wanggs62@yeah.net

Mathematics Subject Classification (2010): Primary 93B07; Secondary 35B35 
This is a kind of stability estimate which shows how the left term $\|u(\cdot, t)\|_{L^{2}(\Omega)}$ depends on the right term $\|u(\cdot, 0)\|_{L^{2}(\Omega)}$. From this point of view, the estimate

$$
\|u(\cdot, T)\|_{L^{2}(\Omega)} \leq C_{(\Omega, n, q, \omega, E, T, a, b)} \int_{D}|u(x, t)| d x d t
$$

where $D=\omega \times E$ with $\omega$ being an open nonempty subset of $\Omega$ and $E$ being a subset of $(0, T]$, shows how the left term $\|u(\cdot, T)\|_{L^{2}(\Omega)}$ depends on the right term $\|u\|_{L^{1}(D)}$. Here and throughout the paper, $C_{(\ldots)}$ denotes a positive constant that only depends on what is enclosed in the brackets. An interesting problem is to ask what kind of E makes (1.3) hold.

When $E=\{T\}$ (or $E=\left\{t_{0}\right\}, t_{0} \in(0, T]$ ), (1.3) does not hold. However, it has been shown (for some potentials $a$ and $b$ ) that

$$
\|u(\cdot, T)\|_{L^{2}(\Omega)} \leq C_{(\Omega, n, q, \omega, T, a, b)}\|u(\cdot, 0)\|_{L^{2}(\Omega)}^{\alpha}\|u(\cdot, T)\|_{L^{2}(\omega)}^{1-\alpha}
$$

for some $\alpha \in(0,1)$. This is a quantitative unique continuation at one point in time. It is a kind of Hölder continuous dependence in the sense of John. We call (1.4) Hölder continuous dependence at one point in time. For studies of unique continuation, we refer the readers to $[\mathrm{BT}],[\mathrm{L}],[\mathrm{K}],[\mathrm{KT}]$ and references therein.

When $E=(0, T)$ (or $E$ is a subinterval of $(0, T)$ ), the estimate (1.3), viewed as a refined observability estimate in the control theory of PDE, has been discussed in many papers (see for instance [LR], [FI], [DZZ]). It is known that the estimate (1.3) holds for a large class of potentials $a$ and $b$ (see [DFGZ]).

The present paper studies the estimate (1.3) when $E$ is a measurable set of $(0, T)$ of positive measure. The main result is as follows.

Theorem 1.1. Let $E \subset(0, T)$ be a measurable set of positive measure. Let $\omega$ be a nonempty open subset of $\Omega$. Then any solution $u$ to (1.1) satisfies the estimate

$$
\|u(\cdot, T)\|_{L^{2}(\Omega)} \leq C_{(\Omega, n, q, \omega, E, T, a, b)} \int_{\omega \times E}|u(x, t)| d x d t .
$$

The key to establishing Theorem 1.1 is the following strategy:

Hölder continuous dependence at one point in time

$$
\Rightarrow \text { observability from a measurable set in time (i.e., (1.5)). }
$$

Two observations follow from the above strategy. First, Hölder continuous dependence at one point in time can imply both an observability estimate and unique continuation for heat equations with lower terms depending on both time and space variables. From this point of view, it has similar functionality to the global Carleman inequality in [FI], Second, the estimate (1.5) can be derived without using either the estimate of LebeauRobbiano type (see [LR]) or the global Carleman inequality. Our method provides a different way from that in [W] to get (1.5) for the case where $a=0, b=0$. We would like to stress the following: The method to derive the analogous estimate in [W] is based on the estimate of Lebeau-Robbiano type and an iterative argument. And it does not work 
for heat equations with lower order terms depending on both time and space variables. Finally, the following facts should be mentioned: The strategy provided in the current paper is partially inspired by $[\mathrm{M}]$; the estimate (1.4) is proved using the technique provided in $[\mathrm{P}],[\mathrm{EFV}]$ and $[\mathrm{PW}]$.

The rest of the paper is organized as follows. In Section 2 we first establish the Hölder continuous dependence at one point in time, and then we prove Theorem 1.1. Section 3 provides some applications of Theorem 1.1 to the bang-bang property for norm and time optimal control problems. In Appendix, the proofs of some results (used in the proof of Theorem 1.1) are given.

\section{Proof of Theorem 1.1}

\subsection{Preliminary results}

The proof of Theorem 1.1 is based on the following two results. The first one is another version of the statement (17.27) in [Li] (see also Lemma 2.1.5 in [F]). We will provide its proof in Appendix for completeness. The proof of the second result will be given in Subsection 2.3 .

Proposition 2.1. Let $E \subset(0, T)$ be a measurable set of positive measure. Let $\ell$ be a density point for $E$. Then for each $z>1$, there exists an $\ell_{1} \in(\ell, T)$ such that the sequence $\left\{\ell_{m}\right\}_{m \geq 1}$, given by

$$
\ell_{m+1}=\ell+\frac{1}{z^{m}}\left(\ell_{1}-\ell\right)
$$

satisfies

$$
\ell_{m}-\ell_{m+1} \leq 3\left|E \cap\left(\ell_{m+1}, \ell_{m}\right)\right| .
$$

To state the second result, we need the following notation. Let

$$
p= \begin{cases}2 n / q & \text { if } n<q \leq 2 n \\ 1 & \text { if } 2 n \leq q\end{cases}
$$

Write

$$
\begin{gathered}
A(T,\|a\|)=\|a\|_{L^{\infty}\left(0, T ; L^{q}(\Omega)\right)}+\left(T+T^{2-p}\right)\|a\|_{L^{\infty}\left(0, T ; L^{q}(\Omega)\right)}^{2}+T^{2}\|a\|_{L^{\infty}\left(0, T ; L^{q}(\Omega)\right)}^{4 /(2-p)}, \\
K(T,\|a\|,\|b\|)=1+A(T,\|a\|)+T\|b\|_{L^{\infty}(\Omega \times(0, T))}^{2}, \\
\beta(r, T,\|b\|)=\frac{1}{r^{2}} e^{2 T\left(1+\|b\|_{L^{\infty}(\Omega \times(0, T))}^{2}\right.} .
\end{gathered}
$$

Proposition 2.2. Let $B_{r}$ be an open ball of radius $r>0$ and contained in $\Omega$. There is a $C=C_{(\Omega, n, q)}$ such that any solution u to (1.1) satisfies

$$
\begin{aligned}
\int_{\Omega}|u(x, L)|^{2} d x \leq & \left(C \int_{B_{r}}|u(x, L)|^{2} d x\right)^{1-\alpha(r, T,\|b\|)} \\
& \times\left(e^{C(K(T,\|a\|,\|b\|)+1 / L)} \int_{\Omega}|u(x, 0)|^{2} d x\right)^{\alpha(r, T,\|b\|)}
\end{aligned}
$$


where $L$ is arbitrarily taken from $(0, T]$, and where

$$
\alpha(r, T,\|b\|)=\frac{C \beta(r, T,\|b\|)}{1+C \beta(r, T,\|b\|)} .
$$

Furthermore, there is a positive constant c (only depending on $\Omega, n$ and $q$ ) such that any solution u to (1.1) satisfies

$$
\begin{aligned}
\left\|u\left(\cdot, t_{2}\right)\right\|_{L^{2}(\Omega)} \leq & \frac{1}{\varepsilon^{\gamma(r, T,\|b\|)}} e^{c\left(K(T,\|a\|,\|b\|)+\frac{1}{t_{2}-t_{1}}\right) \beta(r, T,\|b\|)}\left\|u\left(\cdot, t_{2}\right)\right\|_{L^{1}\left(B_{r}\right)} \\
& +\varepsilon\left\|u\left(\cdot, t_{1}\right)\right\|_{L^{2}(\Omega)} \quad \forall \varepsilon>0,
\end{aligned}
$$

where $t_{1}$ and $t_{2}$ are arbitrarily taken such that $0 \leq t_{1}<t_{2} \leq T$, and where

$$
\gamma(r, T,\|b\|)=C \beta(r / 2, T,\|b\|)(1+n / 2)+n / 2 .
$$

\subsection{Proof of Theorem 1.1}

Write $B_{r}$ for an open ball of radius $r>0$ and contained in $\omega$. Let $\ell$ be a density point for $E$. Let $\left\{\ell_{m}\right\}_{m \geq 1}$ be the sequence provided by Proposition 2.1 with $z=$ $\sqrt{(\gamma+2) /(\gamma+1)}$, where $\gamma$ is given by (2.1.5). Let $t \in\left(\ell_{m+1}, \ell_{m}\right]$. Then we apply (2.1.4) in Proposition 2.2, where $t_{2}=t$ and $t_{1}=\ell_{m+2}$, to get

$$
\begin{aligned}
\|u(\cdot, t)\|_{L^{2}(\Omega)} \leq & \varepsilon^{-\gamma} e^{c\left(K(T,\|a\|,\|b\|)+\frac{1}{t-\ell_{m+2}}\right) \beta(r, T,\|b\|)}\|u(\cdot, t)\|_{L^{1}\left(B_{r}\right)} \\
& +\varepsilon\left\|u\left(\cdot, \ell_{m+2}\right)\right\|_{L^{2}(\Omega)} \quad \forall \varepsilon>0 .
\end{aligned}
$$

Since it follows from (1.2) that

$$
\left\|u\left(\cdot, \ell_{m}\right)\right\|_{L^{2}(\Omega)} \leq e^{C_{0} T\left[\|a\|_{L^{\infty}\left(0, T, L^{q}(\Omega)\right)}^{2}+\|b\|_{L^{\infty}(\Omega \times(0, T))}^{2}\right.}\|u(\cdot, t)\|_{L^{2}(\Omega)},
$$

we integrate (2.2.1) over $E \cap\left(\ell_{m+1}, \ell_{m}\right)$ to get

$$
\begin{gathered}
\left|E \cap\left(\ell_{m+1}, \ell_{m}\right)\right| e^{-C_{0} T\left[\|a\|_{L^{\infty}\left(0, T, L^{q}(\Omega)\right)}^{2}+\|b\|_{L^{\infty}(\Omega \times(0, T))}^{2}\right.}\left\|u\left(\cdot, \ell_{m}\right)\right\|_{L^{2}(\Omega)} \\
\leq \varepsilon^{-\gamma} e^{c\left(K(T,\|a\|,\|b\|)+\frac{1}{\ell_{m+1}-\ell_{m+2}}\right) \beta(r, T,\|b\|)} \int_{E \cap\left(\ell_{m+1}, \ell_{m}\right)}\|u(\cdot, t)\|_{L^{1}\left(B_{r}\right)} d t \\
\quad+\left|E \cap\left(\ell_{m+1}, \ell_{m}\right)\right| \varepsilon\left\|u\left(\cdot, \ell_{m+2}\right)\right\|_{L^{2}(\Omega)} \quad \forall \varepsilon>0 .
\end{gathered}
$$

This, along with (2.1.1) and (2.1.2), indicates that there is a positive constant $d$ (only depending on $\Omega, n$ and $q$ ) such that 


$$
\begin{aligned}
\left\|u\left(\cdot, \ell_{m}\right)\right\|_{L^{2}(\Omega)} & \\
\leq & \varepsilon^{-\gamma} e^{d K(T,\|a\|,\|b\|) \beta(r, T,\|b\|)}\left[\frac{1}{\ell_{1}-\ell} \frac{z^{m}}{z-1}\right] e^{d \beta(r, T,\|b\|)\left[\frac{1}{\ell_{1}-\ell} \frac{z^{m+1}}{z-1}\right]} \\
& \times \int_{E \cap\left(\ell_{m+1}, \ell_{m}\right)}\|u(\cdot, t)\|_{L^{1}\left(B_{r}\right)} d t+\varepsilon\left\|u\left(\cdot, \ell_{m+2}\right)\right\|_{L^{2}(\Omega)} \\
\leq & \varepsilon^{-\gamma} e^{d K(T,\|a\|,\|b\|) \beta(r, T,\|b\|)} e^{(1+d \beta(r, T,\|b\|))\left[\frac{1}{\ell_{1}-\ell} \frac{z^{m+1}}{z-1}\right]} \int_{E \cap\left(\ell_{m+1}, \ell_{m}\right)}\|u(\cdot, t)\|_{L^{1}\left(B_{r}\right)} d t \\
& +\varepsilon\left\|u\left(\cdot, \ell_{m+2}\right)\right\|_{L^{2}(\Omega)} \quad \forall \varepsilon>0,
\end{aligned}
$$

that is,

$$
\begin{aligned}
& \varepsilon^{\gamma} e^{-\eta z^{m+2}}\left\|u\left(\cdot, \ell_{m}\right)\right\|_{L^{2}(\Omega)}-\varepsilon^{\gamma+1} e^{-\eta z^{m+2}}\left\|u\left(\cdot, \ell_{m+2}\right)\right\|_{L^{2}(\Omega)} \\
& \leq e^{d K(T,\|a\|,\|b\|) \beta(r, T,\|b\|)} \int_{E \cap\left(\ell_{m+1}, \ell_{m}\right)}\|u(\cdot, t)\|_{L^{1}\left(B_{r}\right)} d t \quad \forall \varepsilon>0,
\end{aligned}
$$

where $\eta=(1+d \beta(r, T,\|b\|))\left[\frac{1}{\ell_{1}-\ell} \frac{1}{z(z-1)}\right]$. By taking $\varepsilon=e^{-\eta z^{m+2}}$ in (2.2.2), and by using the fact that $(\gamma+1) z^{2}=\gamma+2$, we obtain

$$
\begin{aligned}
& e^{-\eta(\gamma+2) z^{m}}\left\|u\left(\cdot, \ell_{m}\right)\right\|_{L^{2}(\Omega)}-e^{-\eta(\gamma+2) z^{m+2}}\left\|u\left(\cdot, \ell_{m+2}\right)\right\|_{L^{2}(\Omega)} \\
& \leq e^{d K(T,\|a\|,\|b\|) \beta(r, T,\|b\|)} \int_{E \cap\left(\ell_{m+1}, \ell_{m}\right)}\|u(\cdot, t)\|_{L^{1}\left(B_{r}\right)} d t .
\end{aligned}
$$

Next, we take $m=2 m^{\prime}$ and then sum (2.2.3) from $m^{\prime}=1$ to infinity to deduce that

$$
\begin{gathered}
\sum_{m^{\prime}=1}^{\infty}\left[e^{-\eta(\gamma+2) z^{2 m^{\prime}}}\left\|u\left(\cdot, \ell_{2 m^{\prime}}\right)\right\|_{L^{2}(\Omega)}-e^{-\eta(\gamma+2) z^{2 m^{\prime}+2}} \| u\left(\cdot, \ell_{\left.2 m^{\prime}+2\right)} \|_{L^{2}(\Omega)}\right]\right. \\
\leq e^{d K(T,\|a\|,\|b\|) \beta(r, T,\|b\|)} \sum_{m^{\prime}=1}^{\infty} \int_{E \cap\left(\ell_{2 m^{\prime}+1}, \ell_{2 m^{\prime}}\right)}\|u(\cdot, t)\|_{L^{1}\left(B_{r}\right)} d t \\
\leq e^{d K(T,\|a\|,\|b\|) \beta(r, T,\|b\|)} \int_{E}\|u(\cdot, t)\|_{L^{1}\left(B_{r}\right)} d t
\end{gathered}
$$

Since $e^{-\eta(\gamma+2) z^{2 m^{\prime}+2}}$ tends to zero as $m^{\prime} \rightarrow+\infty$, we have

$$
\begin{array}{r}
\sum_{m^{\prime}=1}^{\infty}\left[e^{-\eta(\gamma+2) z^{2 m^{\prime}}}\left\|u\left(\cdot, \ell_{2 m^{\prime}}\right)\right\|_{L^{2}(\Omega)}-e^{-\eta(\gamma+2) z^{2 m^{\prime}+2}}\left\|u\left(\cdot, \ell_{2 m^{\prime}+2}\right)\right\|_{L^{2}(\Omega)}\right] \\
=e^{-\eta(\gamma+2) z^{2}}\left\|u\left(\cdot, \ell_{2}\right)\right\|_{L^{2}(\Omega)} .
\end{array}
$$


Moreover, one can easily check that

$$
\begin{aligned}
\eta(\gamma+2) z^{2} & =(1+d \beta(r, T,\|b\|))\left[\frac{1}{\ell_{1}-\ell}\right](\gamma+2) \sqrt{\gamma+2}(\sqrt{\gamma+2}+\sqrt{\gamma+1}) \\
& =C_{(\Omega, n, q)} \frac{1}{\ell_{1}-\ell}[\beta(r, T,\|b\|)]^{3} .
\end{aligned}
$$

Now, it follows from (2.2.4)-(2.2.6) that

$$
\left\|u\left(\cdot, \ell_{2}\right)\right\|_{L^{2}(\Omega)} \leq e^{C_{(\Omega, n, q)} \frac{1}{\ell_{1}-\ell}[\beta(r, T,\|b\|)]^{3}} e^{d K(T,\|a\|,\|b\|) \beta(r, T,\|b\|)} \int_{E}\|u(\cdot, t)\|_{L^{1}\left(B_{r}\right)} d t .
$$

This, along with the fact that

$$
\|u(\cdot, T)\|_{L^{2}(\Omega)} \leq e^{C_{0} T\left[\|a\|_{L^{\infty}\left(0, T, L^{q}(\Omega)\right)}^{2}+\|b\|_{L^{\infty}(\Omega \times(0, T))}^{2}\right.}\left\|u\left(\cdot, \ell_{2}\right)\right\|_{L^{2}(\Omega)},
$$

indicates that

$$
\begin{aligned}
& \|u(\cdot, T)\|_{L^{2}(\Omega)} \\
& \quad \leq e^{\left(C_{0}+d \beta(r, T,\|b\|)\right) K(T,\|a\|,\|b\|)} e^{C_{(\Omega, n, q)} \frac{1}{\ell_{1}-\ell}[\beta(r, T,\|b\|)]^{3}} \int_{E}\|u(\cdot, t)\|_{L^{1}\left(B_{r}\right)} d t .
\end{aligned}
$$

This leads to the desired results and completes the proof of Theorem 1.1.

\subsection{Proof of Proposition 2.2}

We begin by introducing two quantities, $G_{\lambda}$ and $N_{\lambda, \varphi}$, as follows. Let $x_{0}$ be the center of $B_{r}$. Let $L \in(0, T]$. For each $\lambda>0$, we define

$$
G_{\lambda}(x, t)=\frac{1}{(L-t+\lambda)^{n / 2}} e^{-\frac{\left|x-x_{0}\right|^{2}}{4(L-t+\lambda)}}, \quad(x, t) \in \mathbb{R}^{n} \times[0, L] .
$$

It is clear that $G_{\lambda}$ is a smooth function and satisfies

$$
\left(\partial_{t}+\Delta\right) G_{\lambda}(x, t)=0, \quad(x, t) \in \mathbb{R}^{n} \times[0, L] .
$$

Moreover,

$$
\begin{aligned}
\frac{1}{2} \frac{d}{d t} \int_{\Omega}|u(x, t)|^{2} G_{\lambda}(x, t) d x+ & \int_{\Omega}|\nabla u(x, t)|^{2} G_{\lambda}(x, t) d x \\
& =\int_{\Omega} u(x, t)\left(\partial_{t}-\Delta\right) u(x, t) G_{\lambda}(x, t) d x
\end{aligned}
$$

for any $t \in(0, L]$. This can be proved by a direct computation. Also it can be derived from the following observation. The quantity

$$
\int_{\Omega}\left(\partial_{t}-\Delta\right)\left(|u(x, t)|^{2}\right) G(x, t) d x+\int_{\Omega}|u(x, t)|^{2}\left(\partial_{t}+\Delta\right) G(x, t) d x,
$$


where $G \in C^{\infty}$, has two expressions,

$$
\int_{\Omega} \frac{d}{d t}\left(|u(x, t)|^{2} G(x, t)\right) d x-\int_{\partial \Omega}\left[\partial_{\nu}\left(|u(x, t)|^{2}\right) G(x, t)-|u(x, t)|^{2} \partial_{\nu} G(x, t)\right] d \sigma
$$

and

$$
\begin{aligned}
2 \int_{\Omega}\left[u(x, t)\left(\partial_{t}-\Delta\right) u(x, t)-|\nabla u(x, t)|^{2}\right] G & (x, t) d x \\
& +\int_{\Omega}|u(x, t)|^{2}\left(\partial_{t}+\Delta\right) G(x, t) d x .
\end{aligned}
$$

Because of (2.3.1) and since $u=0$ on $\partial \Omega$, (2.3.2) follows from the above two expressions with $G=G_{\lambda}$.

Next, we define, for each $\lambda>0$ and each $\varphi$ such that $\varphi \in C\left([\tau, L] ; H^{1}(\Omega)\right)$ for any $\tau \in(0, L)$,

$$
N_{\lambda, \varphi}(t)=\frac{\int_{\Omega}|\nabla \varphi(x, t)|^{2} G_{\lambda}(x, t) d x}{\int_{\Omega}|\varphi(x, t)|^{2} G_{\lambda}(x, t) d x}
$$

where $t$ is in the set $\left\{t \in(0, L] ; \varphi(\cdot, t) \neq 0\right.$ in $\left.L^{2}(\Omega)\right\}$.

Proof of (2.1.3) in Proposition 2.2. The first step to prove (2.1.3) is to estimate $\frac{d}{d t} N_{\lambda, u}(t)$. The desired estimate is a consequence of the following lemma.

Lemma 2.3. Let $\left(\varphi_{0}, g\right) \in L^{2}(\Omega) \times L^{2}(\Omega \times(0, L))$ and $\varphi=\varphi(x, t)$ be the solution of

$$
\begin{cases}\partial_{t} \varphi-\Delta \varphi=g & \text { in } \Omega \times(0, L) \\ \varphi=0 & \text { on } \partial \Omega \times(0, L), \\ \varphi(\cdot, 0)=\varphi_{0} . & \end{cases}
$$

Then on the set $\left\{t \in(0, L]: \varphi(\cdot, t) \neq 0\right.$ in $\left.L^{2}(\Omega)\right\}$, the function $t \mapsto N_{\lambda, \varphi}(t)$ is differentiable. Furthermore,

$$
\frac{d}{d t} N_{\lambda, \varphi}(t) \leq \frac{1}{L-t+\lambda} N_{\lambda, \varphi}(t)+\frac{\int_{\Omega}|g(x, t)|^{2} G_{\lambda}(x, t) d x}{\int_{\Omega}|\varphi(x, t)|^{2} G_{\lambda}(x, t) d x} .
$$

Lemma 2.3 is a direct consequence of estimate (3.26) in [PW]. We omit the proof.

The second step to prove (2.1.3) is to estimate $\lambda N_{\lambda, u}(L)$ by making use of (2.3.3) and (2.3.2). The desired estimate is stated in the following lemma.

Lemma 2.4. There exists a $C_{(\Omega, n, q)}$ such that any nontrivial solution u to (1.1) satisfies

$$
\begin{aligned}
\lambda N_{\lambda, u}(L)+n / 4 & \leq 8(\lambda / L+n) e^{2 L\left(1+\|b\|_{L^{\infty}(\Omega \times(0, L))}^{2}\right)} \\
& \times \log \left[e^{\left(1+\left(C_{(\Omega, n, q)}+C_{0}\right)\left[A(L,\|a\|)+L\|b\|_{L^{\infty}(\Omega \times(0, L))}^{2}\right]+\frac{m_{0}}{2 L}\right)} \frac{\int_{\Omega}|u(x, 0)|^{2} d x}{\int_{\Omega}|u(x, L)|^{2} d x}\right],
\end{aligned}
$$

where $m_{0}=\sup _{x \in \Omega}\left|x-x_{0}\right|^{2}$ and $C_{0}$ is given in (1.2). 
Proof of Lemma 2.4. Clearly, the solution $u$ to (1.1) has the property that $u \in$ $L^{2}\left(\tau, T ; H^{2} \cap H_{0}^{1}(\Omega)\right) \cap C\left([\tau, T] ; H_{0}^{1}(\Omega)\right)$ and $\partial_{t} u \in L^{2}\left(\tau, T ; L^{2}(\Omega)\right)$ for any $\tau \in$ $(0, L)$. One can easily check that $N_{\lambda, u}(t)$ is well-defined for any $t \in(0, L]$. We carry out the rest of the proof in three steps as follows.

Step 1. We claim that for any $t \in(0, L]$,

$$
\begin{aligned}
& \frac{\lambda}{L+\lambda} e^{-2 L\left(1+\|b\|_{L^{\infty}(\Omega \times(0, L))}^{2}\right)} N_{\lambda, u}(L) \\
& \quad \leq N_{\lambda, u}(t)+C_{(\Omega, n, q)} L\|a\|_{L^{\infty}\left(0, L ; L^{q}(\Omega)\right)}^{4 /(2-p)}+C_{(\Omega, n, q)} \frac{1}{L^{p-1}}\|a\|_{L^{\infty}\left(0, L ; L^{q}(\Omega)\right)}^{2} .
\end{aligned}
$$

To see this, we apply Lemma 2.3 to $\left(\varphi_{0}, g\right)=(u(\cdot, 0),-a u-b \cdot \nabla u)$ and use the CauchySchwarz inequality to get

$$
\begin{aligned}
\frac{d}{d t} N_{\lambda, u}(t) \leq & \frac{1}{L-t+\lambda} N_{\lambda, u}(t) \\
& +2 \frac{\int_{\Omega}|a u(x, t)|^{2} G_{\lambda}(x, t) d x}{\int_{\Omega}|u(x, t)|^{2} G_{\lambda}(x, t) d x}+2\|b\|_{L^{\infty}(\Omega \times(0, L))}^{2} N_{\lambda, u}(t) .
\end{aligned}
$$

By (A.2.1) in Appendix,

$$
\begin{aligned}
& \int_{\Omega}|a(x, t) u(x, t)|^{2} G_{\lambda}(x, t) d x \leq N_{\lambda, u}(t) \int_{\Omega}|u(x, t)|^{2} G_{\lambda}(x, t) d x \\
& +C_{(\Omega, n, q)}\left(\|a\|_{L^{\infty}\left(0, L ; L^{q}(\Omega)\right)}^{4 /(2-p)}+\frac{\|a\|_{L^{\infty}\left(0, L ; L^{q}(\Omega)\right)}^{2}}{(L-t+\lambda)^{p}}\right) \int_{\Omega}|u(x, t)|^{2} G_{\lambda}(x, t) d x .
\end{aligned}
$$

It follows from (2.3.5) and (2.3.6) that

$$
\begin{aligned}
\frac{d}{d t}[(L-t+ & \lambda) e^{-2 t\left(1+\|b\|_{L^{\infty}(\Omega \times(0, L))}^{2} N_{\lambda, u}(t)\right]} \\
\leq & C_{(\Omega, n, q)}\|a\|_{L^{\infty}\left(0, L ; L^{q}(\Omega)\right)}^{4 /(2-p)}(L-t+\lambda) e^{-2 t\left(1+\|b\|_{L^{\infty}(\Omega \times(0, L))}^{2}\right)} \\
& +C_{(\Omega, n, q)}\|a\|_{L^{\infty}\left(0, L ; L^{q}(\Omega)\right)}^{2} \frac{1}{(L-t+\lambda)^{p-1}} e^{-2 t\left(1+\|b\|_{L^{\infty}(\Omega \times(0, L))}^{2}\right)}
\end{aligned}
$$

Integrating it over $[t, L]$ with $t \in(0, L)$, after some simple computations, we get (2.3.4).

Step 2. We claim that for any $t \in(0, L / 2]$,

$$
\begin{aligned}
& \frac{d}{d t} \int_{\Omega}|u(x, t)|^{2} G_{\lambda}(x, t) d x+\frac{1}{2} N_{\lambda, u}(t) \int_{\Omega}|u(x, t)|^{2} G_{\lambda}(x, t) d x \\
& \leq\left(C_{(\Omega, n, q)}\|a\|_{L^{\infty}\left(0, L ; L^{q}(\Omega)\right)}^{2}+\|b\|_{L^{\infty}(\Omega \times(0, L))}^{2}\right) \int_{\Omega}|u(x, t)|^{2} G_{\lambda}(x, t) d x \\
& \quad+C_{(\Omega, n, q)} \frac{1}{L}\|a\|_{L^{\infty}\left(0, L ; L^{q}(\Omega)\right)} \int_{\Omega}|u(x, t)|^{2} G_{\lambda}(x, t) d x .
\end{aligned}
$$


For this purpose, we first observe that (2.3.2) is equivalent to the following equality:

$$
\begin{aligned}
\frac{d}{d t} \int_{\Omega}|u(x, t)|^{2} G_{\lambda}(x, t) d x+2 N_{\lambda, u}(t) & \int_{\Omega}|u(x, t)|^{2} G_{\lambda}(x, t) d x \\
= & 2 \int_{\Omega} u(x, t)\left(\partial_{t}-\Delta\right) u(x, t) G_{\lambda}(x, t) d x,
\end{aligned}
$$

for any $t \in(0, L]$. From this and the Cauchy-Schwarz inequality, it follows that

$$
\begin{aligned}
\frac{d}{d t} \int_{\Omega}|u(x, t)|^{2} G_{\lambda}(x, t) d x & +N_{\lambda, u}(t) \int_{\Omega}|u(x, t)|^{2} G_{\lambda}(x, t) d x \\
\leq & 2 \int_{\Omega}|a(x, t)||u(x, t)|^{2} G_{\lambda}(x, t) d x \\
& +\|b\|_{L^{\infty}(\Omega \times(0, L))}^{2} \int_{\Omega}|u(x, t)|^{2} G_{\lambda}(x, t) d x
\end{aligned}
$$

By (A.2.2) in Appendix,

$$
\begin{aligned}
& \int_{\Omega} a(x, t)|u(x, t)|^{2} G_{\lambda}(x, t) d x \leq \frac{1}{4} \int_{\Omega}|\nabla u(x, t)|^{2} G_{\lambda}(x, t) d x \\
& +C_{(\Omega, n, q)}\|a\|_{L^{\infty}\left(0, L ; L^{q}(\Omega)\right)}\left(\|a\|_{L^{\infty}\left(0, L ; L^{q}(\Omega)\right)}+\frac{1}{L-t+\lambda}\right) \int_{\Omega}|u(x, t)|^{2} G_{\lambda}(x, t) d x .
\end{aligned}
$$

We directly get (2.3.7) from (2.3.8) and (2.3.9).

Step 3: Conclusion. By (2.3.4) and (2.3.7), we deduce that for any $t \in(0, L / 2]$,

$$
\begin{aligned}
\frac{d}{d t} \int_{\Omega}|u(x, t)|^{2} G_{\lambda}(x, t) d x & \quad+\frac{1}{2} \frac{\lambda}{L+\lambda} e^{-2 L\left(1+\|b\|_{L^{\infty}(\Omega \times(0, L))}^{2}\right.} N_{\lambda, u}(L) \int_{\Omega}|u(x, t)|^{2} G_{\lambda}(x, t) d x \\
\leq & \left(C_{(\Omega, n, q)}\|a\|_{L^{\infty}\left(0, L ; L^{q}(\Omega)\right)}^{2}+\|b\|_{L^{\infty}(\Omega \times(0, L))}^{2}\right) \int_{\Omega}|u(x, t)|^{2} G_{\lambda}(x, t) d x \\
& +C_{(\Omega, n, q)} \frac{1}{L}\|a\|_{L^{\infty}\left(0, L ; L^{q}(\Omega)\right)} \int_{\Omega}|u(x, t)|^{2} G_{\lambda}(x, t) d x \\
& +C_{(\Omega, n, q)}\left[L\|a\|_{L^{\infty}\left(0, L ; L^{q}(\Omega)\right)}^{4 /(2-p)}+\frac{1}{L^{p-1}}\|a\|_{L^{\infty}\left(0, L ; L^{q}(\Omega)\right)}^{2}\right] \\
& \times \int_{\Omega}|u(x, t)|^{2} G_{\lambda}(x, t) d x .
\end{aligned}
$$

Recall that

$$
\begin{aligned}
\frac{1}{L} A(L,\|a\|)= & \|a\|_{L^{\infty}\left(0, L ; L^{q}(\Omega)\right)}^{2}+\frac{1}{L}\|a\|_{L^{\infty}\left(0, L ; L^{q}(\Omega)\right)} \\
& \left.+L\|a\|_{L^{\infty}\left(0, L ; L^{q}(\Omega)\right)}\right)^{4 /(2-p)}+\frac{1}{L^{p-1}}\|a\|_{L^{\infty}\left(0, L ; L^{q}(\Omega)\right)}^{2}
\end{aligned}
$$


This, together with (2.3.10), gives

$$
\begin{aligned}
\frac{d}{d t} \int_{\Omega}|u(x, t)|^{2} G_{\lambda}(x, t) d x & \\
& +\frac{1}{2} \frac{\lambda}{L+\lambda} e^{-2 L\left(1+\|b\|_{L^{\infty}(\Omega \times(0, L))}^{2}\right.} N_{\lambda, u}(L) \int_{\Omega}|u(x, t)|^{2} G_{\lambda}(x, t) d x \\
\leq & \left(C_{(\Omega, n, q)} \frac{1}{L} A(L,\|a\|)+\|b\|_{L^{\infty}(\Omega \times(0, L))}^{2}\right) \int_{\Omega}|u(x, t)|^{2} G_{\lambda}(x, t) d x
\end{aligned}
$$

From this, we deduce that for any $t \in(0, L / 2]$,

$$
\begin{aligned}
\int_{\Omega}|u(x, t)|^{2} G_{\lambda}(x, t) d x \leq & \exp \left(-t\left[\frac{1}{2} \frac{\lambda}{L+\lambda} e^{-2 L\left(1+\|b\|_{L^{\infty}(\Omega \times(0, L))}^{2}\right)} N_{\lambda}(u, L)\right]\right) \\
& \times \exp \left(t\left[C_{(\Omega, n, q)} \frac{1}{L} A(L,\|a\|)+\|b\|_{L^{\infty}(\Omega \times(0, L))}^{2}\right]\right) \\
& \times \int_{\Omega}|u(x, 0)|^{2} G_{\lambda}(x, 0) d x .
\end{aligned}
$$

Taking $t=L / 2$ in the above, we see that

$$
\begin{aligned}
& \frac{1}{(L / 2+\lambda)^{n / 2}} \int_{\Omega}|u(x, L / 2)|^{2} e^{-\frac{\left|x-x_{0}\right|^{2}}{4(L / 2+\lambda)}} d x \\
& \leq \exp \left(-\left[\frac{\lambda L}{4(L+\lambda)} e^{-2 L\left(1+\|b\|_{L^{\infty}(\Omega \times(0, L))}^{2}\right.} N_{\lambda}(u, L)\right]\right) \\
& \quad \times \exp \left(C_{(\Omega, n, q)} A(L,\|a\|)+L\|b\|_{L^{\infty}(\Omega \times(0, L))}^{2}\right) \frac{1}{(L+\lambda)^{n / 2}} \int_{\Omega}|u(x, 0)|^{2} e^{-\frac{\left|x-x_{0}\right|^{2}}{4(L+\lambda)}} d x .
\end{aligned}
$$

On the other hand, it is clear that

$$
\begin{aligned}
& \int_{\Omega}|u(x, L)|^{2} d x \leq e^{C_{0} L\left(\|a\|_{L^{\infty}\left(0, L ; L^{q}(\Omega)\right)}^{2}+\|b\|_{L^{\infty}(\Omega \times(0, L))}^{2}\right)} \int_{\Omega}|u(x, L / 2)|^{2} d x
\end{aligned}
$$

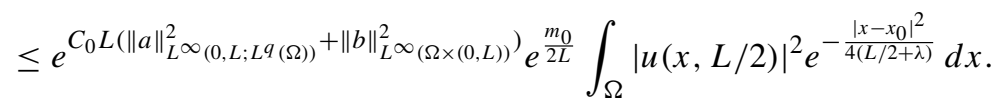

This, together with (2.3.11), yields

$$
\begin{aligned}
\int_{\Omega}|u(x, L)|^{2} d x \leq & \exp \left(-\left[\frac{\lambda L}{4(L+\lambda)} e^{-2 L\left(1+\|b\|_{L^{\infty}(\Omega \times(0, L))}^{2}\right)} N_{\lambda}(u, L)\right]\right) \\
& \times \exp \left(\left(C_{(\Omega, n, q)}+C_{0}\right)\left[A(L,\|a\|)+L\|b\|_{L^{\infty}(\Omega \times(0, L))}^{2}\right]+\frac{m_{0}}{2 L}\right) \\
& \times \int_{\Omega}|u(x, 0)|^{2} d x,
\end{aligned}
$$


from which it follows that

$$
\begin{aligned}
& \lambda N_{\lambda, u}(L) \leq 4(\lambda / L+1) e^{2 L\left(1+\|b\|_{L^{\infty}(\Omega \times(0, L))}^{2}\right)} \\
& \times \log \left[e^{\left(\left(C_{(\Omega, n, q)}+C_{0}\right)\left[A(L,\|a\|)+L\|b\|_{L^{\infty}(\Omega \times(0, L))}^{2}\right]+\frac{m_{0}}{2 L}\right)} \frac{\int_{\Omega}|u(x, 0)|^{2} d x}{\int_{\Omega}|u(x, L)|^{2} d x}\right] .
\end{aligned}
$$

Clearly,

$$
\frac{n}{4} \leq \frac{n}{4} \log \left[e^{\left(1+\left(C_{(\Omega, n, q)}+C_{0}\right)\left[A(L,\|a\|)+L\|b\|_{L^{\infty}(\Omega \times(0, L))}^{2}\right]+\frac{m}{2 L}\right)} \frac{\int_{\Omega}|u(x, 0)|^{2} d x}{\int_{\Omega}|u(x, L)|^{2} d x}\right] .
$$

Now, the desired estimate in Lemma 2.4 follows immediately from (2.3.12) and (2.3.13). This completes the proof of Lemma 2.4.

The third step to prove (2.1.3) is to get an estimate of $\int_{\Omega}|u(x, L)|^{2} e^{-\left|x-x_{0}\right|^{2} /(4 \lambda)} d x$ in terms of $\int_{B_{r}}|u(x, L)|^{2} e^{-\left|x-x_{0}\right|^{2} /(4 \lambda)} d x$. It is a consequence of the following lemma.

Lemma 2.5. For any nontrivial $f \in H^{1}(\Omega)$ and any $\lambda>0$,

$$
\begin{aligned}
\int_{\Omega}|f(x)|^{2} e^{-\frac{\left|x-x_{0}\right|^{2}}{4 \lambda}} d x \leq & \int_{B_{r}}|f(x)|^{2} e^{-\frac{\left|x-x_{0}\right|^{2}}{4 \lambda}} d x \\
& +\frac{16 \lambda}{r^{2}}\left(\lambda N_{\lambda, f}(L)+n / 4\right) \int_{\Omega}|f(x)|^{2} e^{-\frac{\left|x-x_{0}\right|^{2}}{4 \lambda}} d x .
\end{aligned}
$$

Proof of Lemma 2.5. We first observe that

$$
\begin{aligned}
\int_{\Omega}|f(x)|^{2} e^{-\frac{\left|x-x_{0}\right|^{2}}{4 \lambda}} d x & \\
& \leq \int_{B_{r}}|f(x)|^{2} e^{-\frac{\left|x-x_{0}\right|^{2}}{4 \lambda}} d x+\int_{\Omega \cap\left\{\left|x-x_{0}\right| \geq r\right\}}|f(x)|^{2} e^{-\frac{\left|x-x_{0}\right|^{2}}{4 \lambda}} d x \\
& \leq \int_{B_{r}}|f(x)|^{2} e^{-\frac{\left|x-x_{0}\right|^{2}}{4 \lambda}} d x+\frac{16 \lambda}{r^{2}} \int_{\Omega} \frac{\left|x-x_{0}\right|^{2}}{16 \lambda}|f(x)|^{2} e^{-\frac{\left|x-x_{0}\right|^{2}}{4 \lambda}} d x
\end{aligned}
$$

Next, we claim that

$$
\begin{aligned}
\int_{\Omega} \frac{\left|x-x_{0}\right|^{2}}{16 \lambda} \mid & \left.f(x)\right|^{2} e^{-\frac{\left|x-x_{0}\right|^{2}}{4 \lambda}} d x \\
& \leq \lambda \int_{\Omega}|\nabla f(x)|^{2} e^{-\frac{\left|x-x_{0}\right|^{2}}{4 \lambda}} d x+\frac{n}{4} \int_{\Omega}|f(x)|^{2} e^{-\frac{\left|x-x_{0}\right|^{2}}{4 \lambda}} d x .
\end{aligned}
$$

When this is at hand, the desired estimate in Lemma 2.5 follows at once from (2.3.14) and (2.3.15). To show (2.3.15), we argue as follows (see also [EFV, p. 211]): 


$$
\begin{aligned}
& \int_{\Omega}\left|x-x_{0}\right|^{2}|f(x)|^{2} e^{-\frac{\left|x-x_{0}\right|^{2}}{4 \lambda}} d x=\int_{\Omega}\left(x-x_{0}\right)|f(x)|^{2} \cdot(-2 \lambda) \nabla e^{-\frac{\left|x-x_{0}\right|^{2}}{4 \lambda}} d x \\
& =-2 \lambda \int_{\partial \Omega}\left(\left(x-x_{0}\right) \cdot v\right)|f(x)|^{2} e^{-\frac{\left|x-x_{0}\right|^{2}}{4 \lambda}} d \sigma+2 \lambda n \int_{\Omega}|f(x)|^{2} e^{-\frac{\left|x-x_{0}\right|^{2}}{4 \lambda}} d x \\
& \quad+4 \lambda \int_{\Omega}\left(x-x_{0}\right) f(x) \cdot \nabla f(x) e^{-\frac{\left|x-x_{0}\right|^{2}}{4 \lambda}} d x \\
& \leq 2 \lambda n \int_{\Omega}|f(x)|^{2} e^{-\frac{\left|x-x_{0}\right|^{2}}{4 \lambda}} d x \\
& \quad+\frac{1}{2} \int_{\Omega} 16 \lambda^{2}|\nabla f(x)|^{2} e^{-\frac{\left|x-x_{0}\right|^{2}}{4 \lambda}} d x+\frac{1}{2} \int_{\Omega}\left|x-x_{0}\right|^{2}|f(x)|^{2} e^{-\frac{\left|x-x_{0}\right|^{2}}{4 \lambda}} d x .
\end{aligned}
$$

In (2.3.16), we use $(-2 \lambda) \nabla e^{-\frac{\left|x-x_{0}\right|^{2}}{4 \lambda}}=\left(x-x_{0}\right) e^{-\frac{\left|x-x_{0}\right|^{2}}{4 \lambda}}$ in the first equality; integration by parts in the second equality; and the Cauchy-Schwarz inequality, along with the assumption that $\Omega$ is convex, in the last inequality. This completes the proof of Lemma 2.5.

The last step to prove (2.1.3) of Proposition 2.2 is to drop the weight function $e^{-\frac{\left|x-x_{0}\right|^{2}}{4 \lambda}}$ in the integrands.

Recall that for any $\tau \in(0, L), u \in L^{2}\left(\tau, T ; H^{2} \cap H_{0}^{1}(\Omega)\right) \cap C\left([\tau, T] ; H_{0}^{1}(\Omega)\right)$ and $\partial_{t} u \in L^{2}\left(\tau, T ; L^{2}(\Omega)\right)$. Without loss of generality, we assume that $u$ is nontrivial so that $N_{\lambda, u}(t)$ is well-defined for any $t \in(0, L]$. We apply Lemma 2.5 where $f=u(\cdot, L)$ to get

$$
\begin{aligned}
\int_{\Omega}|u(x, L)|^{2} e^{-\frac{\left|x-x_{0}\right|^{2}}{4 \lambda}} d x & \leq \int_{B_{r}}|u(x, L)|^{2} e^{-\frac{\left|x-x_{0}\right|^{2}}{4 \lambda}} d x \\
& +\frac{16 \lambda}{r^{2}}\left(\lambda N_{\lambda, u}(L)+n / 4\right) \int_{\Omega}|u(x, L)|^{2} e^{-\frac{\left|x-x_{0}\right|^{2}}{4 \lambda}} d x .
\end{aligned}
$$

By Lemma 2.4,

$$
\lambda N_{\lambda, u}(L)+n / 4 \leq \frac{1}{16}(\lambda / L+n) Z_{u},
$$

where

$$
\begin{aligned}
& Z_{u}=16 \times 8 e^{2 L\left(1+\|b\|_{L_{(\Omega \times(0, L))}^{2}}\right)} \\
& \left.\times \log \left[e^{\left(1+\left(C_{(\Omega, n, q)}+C_{0}\right)\left[A(L,\|a\|)+L\|b\|_{L^{\infty}(\Omega \times(0, L)}^{2}\right)\right.}+\frac{m_{0}}{2 L}\right) \frac{\int_{\Omega}|u(x, 0)|^{2} d x}{\int_{\Omega}|u(x, L)|^{2} d x}\right] .
\end{aligned}
$$

Combining (2.3.17) and (2.3.18), we see that for any $\lambda>0$,

$$
\begin{aligned}
\int_{\Omega}|u(x, L)|^{2} e^{-\frac{\left|x-x_{0}\right|^{2}}{4 \lambda}} d x \leq & \int_{B_{r}}|u(x, L)|^{2} e^{-\frac{\left|x-x_{0}\right|^{2}}{4 \lambda}} d x \\
& +\frac{\lambda}{r^{2}}(\lambda / L+n) Z_{u} \int_{\Omega}|u(x, L)|^{2} e^{-\frac{\left|x-x_{0}\right|^{2}}{4 \lambda}} d x .
\end{aligned}
$$

We take

$$
\lambda=\frac{1}{2}\left(-n L+\sqrt{n^{2} L^{2}+2 L r^{2} / Z_{u}}\right) .
$$


Clearly it solves

$$
\frac{\lambda}{r^{2}}(\lambda / L+n) Z_{u}=\frac{1}{2} .
$$

Then it follows from (2.3.20) and (2.3.21) that

$$
\int_{\Omega}|u(x, L)|^{2} d x \leq 2 e^{\frac{m_{0}}{4 \lambda}} \int_{B_{r}}|u(x, L)|^{2} d x,
$$

where $m_{0}$ is given by Lemma 2.4.

On the other hand, we have

$$
e^{\frac{m_{0}}{4 \lambda}} \leq e^{\frac{(n+1) m_{0}}{2} \frac{Z_{u}}{r^{2}}}
$$

because

$$
\begin{aligned}
\frac{1}{\lambda} & =\frac{2}{-n L+\sqrt{n^{2} L^{2}+2 L r^{2} / Z_{u}}}=\frac{Z_{u}}{L r^{2}}\left(n L+\sqrt{n^{2} L^{2}+2 L r^{2} / Z_{u}}\right) \\
& \leq \frac{Z_{u}}{L r^{2}}\left(n L+\sqrt{n^{2} L^{2}+4 L^{2} r^{2} / m_{0}}\right) \leq \frac{Z_{u}}{r^{2}}\left(n+\sqrt{n^{2}+4}\right) .
\end{aligned}
$$

In the first inequality above, we used that $Z_{u}>\frac{m_{0}}{2 L}$. Now it follows from (2.3.22) and (2.3.23) that

$$
\int_{\Omega}|u(x, L)|^{2} d x \leq 2 e^{\frac{(n+1) m_{0}}{2} \frac{Z_{u}}{r^{2}}} \int_{B_{r}}|u(x, L)|^{2} d x .
$$

Next, by (2.3.19), there is a $C=C_{(\Omega, n, q)}>2$ such that

$$
\frac{(n+1) m_{0}}{2} \frac{Z_{u}}{r^{2}} \leq C \beta(r, T,\|b\|) \log \left[e^{C(K(T,\|a\|,\|b\|)+1 / L)} \frac{\int_{\Omega}|u(x, 0)|^{2} d x}{\int_{\Omega}|u(x, L)|^{2} d x}\right] .
$$

This, together with (2.3.24), yields that

$$
\begin{aligned}
& \int_{\Omega}|u(x, L)|^{2} d x \\
& \quad \leq 2\left[e^{C(K(T,\|a\|,\|b\|)+1 / L)} \frac{\int_{\Omega}|u(x, 0)|^{2} d x}{\int_{\Omega}|u(x, L)|^{2} d x}\right]^{C \beta(r, T,\|b\|)} \int_{B_{r}}|u(x, L)|^{2} d x .
\end{aligned}
$$

In summary, we conclude that

$$
\begin{aligned}
\int_{\Omega}|u(x, L)|^{2} d x \leq & \left(2 \int_{B_{r}}|u(x, L)|^{2} d x\right)^{\frac{1}{1+C \beta(r, T,\|b\|)}} \\
& \times\left(e^{C(K(T,\|a\|,\|b\|)+1 / L)} \int_{\Omega}|u(x, 0)|^{2} d x\right)^{\frac{C \beta(r, T,\|b\|)}{1+C \beta(r, T,\|b\|)}},
\end{aligned}
$$

which leads to (2.1.3). 
Proof of (2.1.4) in Proposition 2.2. Let $0 \leq t_{1}<t_{2} \leq T$. The estimate (2.1.3) implies that

$$
\begin{aligned}
\left\|u\left(\cdot, t_{2}\right)\right\|_{L^{2}(\Omega)} \leq & \left(\sqrt{C}\left\|u\left(\cdot, t_{2}\right)\right\|_{L^{2}\left(B_{r / 2}\right)}\right)^{1-\alpha(r / 2, T,\|b\|)} \\
& \times\left(e^{C\left(K(T,\|a\|,\|b\|)+\frac{1}{t_{2}-t_{1}}\right)}\left\|u\left(\cdot, t_{1}\right)\right\|_{L^{2}(\Omega)}\right)^{\alpha(r / 2, T,\|b\|)} .
\end{aligned}
$$

On the other hand, by the Nash inequality and Poincare inequality, there exists $c>0$ (depending only on $\Omega$ and $n$ ) such that

$$
\left\|u\left(\cdot, t_{2}\right)\right\|_{L^{2}\left(B_{r / 2}\right)}^{1+2 / n} \leq \frac{c}{r}\left\|u\left(\cdot, t_{2}\right)\right\|_{L^{1}\left(B_{r}\right)}^{2 / n}\left\|\nabla u\left(\cdot, t_{2}\right)\right\|_{L^{2}(\Omega)} .
$$

It follows from the standard energy method that

$$
\left\|\nabla u\left(\cdot, t_{2}\right)\right\|_{L^{2}(\Omega)} \leq \frac{1}{\left(t_{2}-t_{1}\right)^{1 / 2}} e^{c\left(1+T\left[\|a\|_{L^{\infty}\left(0, T ; L_{(\Omega))}\right.}^{2}+\|b\|_{L^{\infty}(\Omega \times(0, T)}^{2}\right]\right)}\left\|u\left(\cdot, t_{1}\right)\right\|_{L^{2}(\Omega)},
$$

where $c>0$ (depending only on $\Omega, n$ and $q$ ). Combining (2.3.25)-(2.3.27), we deduce that there is a positive constant $d$ (only depending on $\Omega, n$ and $q$ ) such that

$$
\begin{aligned}
\left\|u\left(\cdot, t_{2}\right)\right\|_{L^{2}(\Omega)} \leq & \left(\frac{1}{r}\right) e^{\frac{1-\alpha(r / 2, T,\|b\|)}{1+2 / n}} e^{d\left(K(T,\|a\|,\|b\|)+\frac{1}{t_{2}-t_{1}}\right)} \\
& \times\left\|u\left(\cdot, t_{2}\right)\right\|_{L^{1}\left(B_{r}\right)}^{\frac{2 / n}{1+2 / n}[1-\alpha(r / 2, T,\|b\|)]}\left\|u\left(\cdot, t_{1}\right)\right\|_{L^{2}(\Omega)}^{1-\frac{2 / n}{1+2 / n}[1-\alpha(r / 2, T,\|b\|)]} .
\end{aligned}
$$

This, together with some simple computations, leads to estimate (2.1.4), and completes the proof of Proposition 2.2.

\section{Applications to bang-bang controls}

Throughout this section, we assume that $a \in L^{\infty}(\Omega \times(0, T)), B \in L^{\infty}(\Omega \times(0, T))^{n}$ with $\operatorname{div} B \in L^{\infty}(\Omega \times(0, T))$ and $y^{0} \in L^{2}(\Omega)$; we let $\omega$ be a nonempty open subset of $\Omega$; and we denote by $1_{\mid X}$ the characteristic function of the set $X$.

Let $\tau \in[0, T)$. Let $E \subset(\tau, T)$ be a measurable set of positive measure. Consider the following parabolic equation:

$$
\begin{cases}\partial_{t} \psi-\Delta \psi+a \psi+B \cdot \nabla \psi=1_{\mid \omega \times(\tau, T)} 1_{\mid E} v & \text { in } \Omega \times(0, T), \\ \psi=0 & \text { on } \partial \Omega \times(0, T), \\ \psi(\cdot, 0)=\psi^{0} & \text { in } \Omega,\end{cases}
$$

where $v \in L^{\infty}(\Omega \times(0, T))$ and $\psi^{0} \in L^{2}(\Omega)$. Then (3.1) admits a unique solution $\psi$ in $C\left([0, T] ; L^{2}(\Omega)\right) \cap L^{2}\left(0, T ; H_{0}^{1}(\Omega)\right)$. The adjoint equation of (3.1) is

$$
\begin{cases}-\partial_{t} \vartheta-\Delta \vartheta+(a-\operatorname{div} B) \vartheta-B \cdot \nabla \vartheta=0 & \text { in } \Omega \times(0, T), \\ \vartheta=0 & \text { on } \partial \Omega \times(0, T), \\ \vartheta(\cdot, T) \in L^{2}(\Omega) . & \end{cases}
$$


By Theorem 1.1, any solution $\vartheta$ to (3.2) satisfies

$$
\|\vartheta(\cdot, 0)\|_{L^{2}(\Omega)} \leq \kappa \int_{\omega \times E}|\vartheta(x, t)| d x d t,
$$

where the constant $\kappa$ is independent of $\vartheta$. This is equivalent to the null-controllability from $E$ : for any $\psi^{0} \in L^{2}(\Omega)$, there is a $v \in L^{\infty}(\Omega \times(0, T))$ with

$$
\|v\|_{L^{\infty}(\Omega \times(0, T))} \leq \kappa\left\|\psi^{0}\right\|_{L^{2}(\Omega)}
$$

such that the corresponding solution $\psi$ to (3.1) satisfies $\psi(\cdot, T)=0$ in $\Omega$ (see e.g. [W]). In general, such a $v$ is not unique.

\subsection{Norm optimal bang-bang control}

Consider the following parabolic equation:

$$
\begin{cases}\partial_{t} y-\Delta y+a y+B \cdot \nabla y=1_{\mid \omega \times(\tau, T)} f & \text { in } \Omega \times(0, T), \\ y=0 & \text { on } \partial \Omega \times(0, T), \\ y(\cdot, 0)=y^{0} & \text { in } \Omega,\end{cases}
$$

where $f \in L^{\infty}\left(0, T ; L^{2}(\Omega)\right)$. Then equation (3.1.1) admits a unique solution $y$ in $C\left([0, T] ; L^{2}(\Omega)\right) \cap L^{2}\left(0, T ; H_{0}^{1}(\Omega)\right)$. Write

$$
\mathcal{F}=\left\{f \in L^{\infty}\left(0, T ; L^{2}(\Omega)\right): y(\cdot, T)=0 \text { in } \Omega\right\},
$$

where $y$ is the solution of (3.1.1) corresponding to $f$.

Theorem 3.1. There is a unique $f^{*} \in \mathcal{F}$ such that

$$
\left\|f^{*}\right\|_{L^{\infty}\left(\tau, T ; L^{2}(\Omega)\right)}=\min _{f \in \mathcal{F}}\|f\|_{L^{\infty}\left(\tau, T ; L^{2}(\Omega)\right)} .
$$

Furthermore, $f^{*}$ has the bang-bang property:

$$
\left\|f^{*}(\cdot, t)\right\|_{L^{2}(\Omega)}=\left\|f^{*}\right\|_{L^{\infty}\left(\tau, T ; L^{2}(\Omega)\right)} \quad \text { for a.e. } t \in(\tau, T) .
$$

Remark 3.2. In the control theory of PDE, the equation (3.1.1) is called a controlled system while $f$ is called a control. $f \in \mathcal{F}$ means that the control $f$ in $L^{\infty}\left(0, T ; L^{2}(\Omega)\right)$ drives the solution $y$ of (3.1.1) from $y^{0}$ to zero at time $T$. The property that $\mathcal{F}$ is nonempty is called the null-controllability for (3.1.1). The quantity

$$
\tilde{M}=\min _{\widetilde{f} \in \mathcal{F}}\|\widetilde{f}\|_{L^{\infty}\left(\tau, T ; L^{2}(\Omega)\right)}
$$

measures the best cost of such controls. The norm optimal control problem (with respect to (3.1.1)) is to ask for a control $f \in \mathcal{F}$ such that $\|f\|_{L^{\infty}\left(\tau, T ; L^{2}(\Omega)\right)}=\widetilde{M}$. Such a control is called a norm optimal control. The norm optimal control problem has the bang-bang property if any norm optimal control $f$ satisfies $\|f(\cdot, t)\|_{L^{2}(\Omega)}=\widetilde{M}$ for a.e. $t \in(\tau, T)$. Theorem 3.1 states that the norm optimal problem has a unique solution and the solution has the bang-bang property. 
Proof of Theorem 3.1. We carry out the proof in three steps.

Step 1: Existence. By the well-known result on the null controllability of parabolic equations (see [DFGZ]), we have $\mathcal{F} \neq \emptyset$. Then by the standard argument of the calculus of variations, we get the existence of a control $f \in \mathcal{F}$ satisfying $\|f\|_{L^{\infty}\left(\tau, T ; L^{2}(\Omega)\right)}=\tilde{M}$.

Step 2: Bang-bang property. We prove that if $f \in \mathcal{F}$ satisfies (3.1.2), then it must satisfy (3.1.3). Seeking a contradiction, we suppose that (3.1.3) does not hold for some $f \in \mathcal{F}$ satisfying (3.1.2). Then there is an $\varepsilon \in(0,1)$ and a measurable set $E \subset(\tau, T)$ of positive measure such that

$$
\|f(\cdot, t)\|_{L^{2}(\Omega)} \leq \tilde{M}-\varepsilon \quad \forall t \in E .
$$

Here $\tilde{M}$ is given by (3.1.4). We claim that for some $\delta \in(0,1)$ there is an $f_{\delta} \in$ $L^{\infty}\left(0, T ; L^{2}(\Omega)\right)$ with

$$
\left\|f_{\delta}\right\|_{L^{\infty}\left(\tau, T ; L^{2}(\Omega)\right)} \leq(1-\delta) \tilde{M}
$$

and a function $y_{\delta}$ with the property that

$$
\begin{cases}\partial_{t} y_{\delta}-\Delta y_{\delta}+a y_{\delta}+B \cdot \nabla y_{\delta}=1_{\mid \omega \times(\tau, T)} f_{\delta} & \text { in } \Omega \times(0, T), \\ y_{\delta}=0 & \text { on } \partial \Omega \times(0, T), \\ y_{\delta}(\cdot, 0)=y^{0} & \text { in } \Omega, \\ y_{\delta}(\cdot, T)=0 & \text { in } \Omega .\end{cases}
$$

The existence of a triplet $\left(\delta, f_{\delta}, y_{\delta}\right)$ that satisfies (3.1.6) and (3.1.7) clearly contradicts the definition of $\widetilde{M}$.

Now, we prove the claim. Let $\delta \in(0,1)$ (to be determined later). By Theorem 1.1 and its equivalence to the null-controllability from $E$, there is a control $v_{\delta} \in L^{\infty}(\Omega \times 0, T)$ such that the solution $\psi_{\delta}$ to

$$
\begin{cases}\partial_{t} \psi_{\delta}-\Delta \psi_{\delta}+a \psi_{\delta}+B \cdot \nabla \psi_{\delta}=1_{\mid \omega \times(\tau, T)} 1_{\mid E} v_{\delta} & \text { in } \Omega \times(0, T), \\ \psi_{\delta}=0 & \text { on } \partial \Omega \times(0, T), \\ \psi_{\delta}(\cdot, 0)=\delta y^{0} & \text { in } \Omega,\end{cases}
$$

satisfies $\psi_{\delta}(\cdot, T)=0$ in $\Omega$. Furthermore, there is a $\kappa>0$ (independent of $\delta$ ) such that

$$
\left\|v_{\delta}\right\|_{L^{\infty}\left(0, T ; L^{2}(\Omega)\right)} \leq|\Omega|^{1 / 2}\left\|v_{\delta}\right\|_{L^{\infty}(\Omega \times(0, T))} \leq \kappa \delta\left\|y^{0}\right\|_{L^{2}(\Omega)} .
$$

Then we define

$$
f_{\delta}=(1-\delta) f+1_{\mid E} v_{\delta} .
$$

By taking $\delta=\varepsilon /\left(\kappa\left\|y^{0}\right\|_{L^{2}(\Omega)}+\varepsilon\right)$, one can easily check that

$$
\left\|f_{\delta}(\cdot, t)\right\|_{L^{2}(\Omega)} \leq(1-\delta) \tilde{M} \quad \text { for a.e. } t \in(\tau, T) .
$$

On the other hand, one can verify that the function $(1-\delta) y+\psi_{\delta}$ satisfies (3.1.7). This, together with (3.1.11), shows the claim.

Step 3: Uniqueness. By the bang-bang property and the parallelogram identity, we can easily check that the control $f \in \mathcal{F}$ satisfying $\|f\|_{L^{\infty}\left(\tau, T ; L^{2}(\Omega)\right)}=\tilde{M}$ is unique (see [F, p. 45]).

This completes the proof. 
Remark 3.3. The problem studied above is indeed a minimal norm control problem. This type of problem has been extensively studied. Here, we would like to mention [Z] where the existence of a bang-bang minimal norm control was proved. The paper [FPZ] also presents the existence of a bang-bang minimal norm control for approximate controllability of the heat equation. However, the uniqueness of the minimal norm control was not proved in either paper.

\subsection{Time optimal bang-bang control}

Consider the parabolic equation

$$
\begin{cases}\partial_{t} y-\Delta y+a y+B \cdot \nabla y=1_{\mid \omega \times(\tau, T)} g & \text { in } \Omega \times(0, T), \\ y=0 & \text { on } \partial \Omega \times(0, T), \\ y(\cdot, 0)=y^{0} & \text { in } \Omega,\end{cases}
$$

where $g \in L^{\infty}\left(0, T ; L^{2}(\Omega)\right)$. Write

$$
\mathcal{G}^{M}=\left\{g \in L^{\infty}\left(0, T ; L^{2}(\Omega)\right):\|g\|_{L^{\infty}\left(0, T ; L^{2}(\Omega)\right)} \leq M\right\},
$$

where $M>0$. We define

$$
\mathcal{P}^{M}=\left\{(\tau, g) \in[0, T) \times \mathcal{G}^{M}: y(\cdot, T)=0 \text { in } \Omega\right\},
$$

where $y$ is the solution of (3.2.1) corresponding to $g$.

Theorem 3.4. Suppose that $\mathcal{P}^{M} \neq \emptyset$. If $\left(\tau^{*}, g^{*}\right) \in \mathcal{P}^{M}$ is such that

$$
\tau^{*} \geq \tau \quad \text { for any pair }(\tau, g) \in \mathcal{P}^{M},
$$

then $g^{*}$ has the bang-bang property:

$$
\left\|g^{*}(\cdot, t)\right\|_{L^{2}(\Omega)}=M \quad \text { for a.e. } t \in\left(\tau^{*}, T\right) .
$$

Furthermore, there is at most one such pair $\left(\tau^{*}, g^{*}\right)$.

Remark 3.5. It may happen that $\mathcal{P}^{M}=\emptyset$. To guarantee that $\mathcal{P}^{M} \neq \emptyset$ for some $T>0$, it is necessary to impose certain conditions on the potentials $a$ and $B$. For instance, it can be checked that each of the following two conditions implies that $\mathcal{P}^{M} \neq \emptyset$ :

- $0 \leq a-\frac{1}{2} \operatorname{div} B+\lambda_{1}$ for a.e. $(x, t) \in \Omega \times(0, T)$;

- $\left\|a-\frac{1}{2} \operatorname{div} B\right\|_{L^{\infty}(\Omega \times(0, T))} \leq \lambda_{1}$.

Here $\lambda_{1}>0$ denotes the first Dirichlet eigenvalue.

Remark 3.6. There is a kind of time optimal control problem whose aim is to delay initiation of active control (in a control constraint set) as late as possible, such that the corresponding solution (of a controlled system) reaches a target by a fixed ending time 
(see e.g. [MS]). In the current study, the controlled system is (3.2.1), where $g$ is viewed as a control; the target is $\{0\} \subset L^{2}(\Omega)$; the ending time is $T$; and the control constraint set is given by (3.2.2), where $M$ is regarded as a bound on controls. $(\tau, g) \in \mathcal{P}^{M}$ means that the control $g$ is not active in $\Omega \times(0, \tau)$ and drives the solution of (3.2.1) from $y^{0}$ to zero at time $T$. The time

$$
\tau^{*}=\max _{(\tau, g) \in \mathcal{P}^{M}} \tau
$$

is called the optimal time; while a control $g^{*}$, with $\left(\tau^{*}, g^{*}\right) \in \mathcal{P}^{M}$, is called a time optimal control. Now from the perspective of the control theory of PDE, Theorem 3.4 states that any time optimal control $g^{*}$ has the bang-bang property: $\left\|g^{*}(\cdot, t)\right\|_{L^{2}(\Omega)}=M$ for a.e. $t \in\left(\tau^{*}, T\right)$. It also shows that the optimal control, if it exists, is unique.

Proof of Theorem 3.4. The uniqueness of the pair $\left(\tau^{*}, g^{*}\right)$ follows directly from the bangbang property (3.2.5) and the parallelogram identity (see [F, p. 45]). Thus, it remains to prove (3.2.5). For contradiction, we suppose that there were a pair $\left(\tau^{*}, g^{*}\right) \in \mathcal{P}^{M}$ satisfying (3.2.4) such that (3.2.5) did not hold. Then there would be an $\varepsilon \in(0,1)$ and a measurable set $\widetilde{E} \subset\left(\tau^{*}, T\right)$ of positive measure such that

$$
\left\|g^{*}(\cdot, t)\right\|_{L^{2}(\Omega)} \leq M-\varepsilon \quad \forall t \in \widetilde{E} .
$$

We claim that there are $\delta \in(0,1)$ and a pair $(y, g)$ with $g \in \mathcal{G}^{M}$ such that

$$
\begin{cases}\partial_{t} y-\Delta y+a y+B \cdot \nabla y=1_{\mid \omega \times\left(\tau^{*}+\delta, T\right)} g & \text { in } \Omega \times(0, T), \\ y=0 & \text { on } \partial \Omega \times(0, T), \\ y(\cdot, 0)=y^{0} & \text { in } \Omega, \\ y(\cdot, T)=0 & \text { in } \Omega .\end{cases}
$$

The existence of such $(\delta, y, g)$ clearly contradicts (3.2.4). To prove the claim, we first observe that there is a $\delta_{0} \in(0,1)$ such that the measurable set

$$
E=\widetilde{E} \cap\left(\tau^{*}+\delta_{0}, T\right)
$$

has a positive measure. Then, it follows from (3.2.6) that

$$
\left\|g^{*}(\cdot, t)\right\|_{L^{2}(\Omega)} \leq M-\varepsilon \quad \forall t \in E .
$$

Let $\delta \in\left(0, \delta_{0}\right)$, to be determined later. By solving the equation

$$
\begin{cases}\partial_{t} z-\Delta z+a z+B \cdot \nabla z=-1_{\mid \omega \times\left(\tau^{*}, \tau^{*}+\delta\right)} g^{*} & \text { in } \Omega \times\left(0, \tau^{*}+\delta\right), \\ z=0 & \text { on } \partial \Omega \times\left(0, \tau^{*}+\delta\right), \\ z(\cdot, 0)=0 & \text { in } \Omega,\end{cases}
$$

we get

$$
\left\|z\left(\cdot, \tau^{*}+\delta\right)\right\|_{L^{2}(\Omega)} \leq c_{0}\left\|g^{*}\right\|_{L^{1}\left(\tau^{*}, \tau^{*}+\delta ; L^{2}(\Omega)\right)} \leq c_{0} M \delta,
$$

where $c_{0}>0$ is independent of $\delta$. 
Next, by Theorem 1.1 and its equivalence to the null-controllability from $E$, there is a control $v \in L^{\infty}\left(\Omega \times\left(\tau^{*}+\delta, T\right)\right)$ such that the solution $\psi$ to the equation

$$
\begin{cases}\partial_{t} \psi-\Delta \psi+a \psi+B \cdot \nabla \psi=1_{\mid \omega \times\left(\tau^{*}+\delta, T\right)} 1_{\mid E} v & \text { in } \Omega \times\left(\tau^{*}+\delta, T\right), \\ \psi=0 & \text { on } \partial \Omega \times\left(\tau^{*}+\delta, T\right), \\ \psi\left(\cdot, \tau^{*}+\delta\right)=z\left(\cdot, \tau^{*}+\delta\right) & \text { in } \Omega,\end{cases}
$$

satisfies $\psi(\cdot, T)=0$ in $\Omega$. Furthermore,

$$
\|v\|_{L^{\infty}\left(\tau^{*}+\delta, T ; L^{2}(\Omega)\right)} \leq|\Omega|^{1 / 2}\|v\|_{L^{\infty}\left(\Omega \times\left(\tau^{*}+\delta, T\right)\right)} \leq \kappa\left\|z\left(\cdot, \tau^{*}+\delta\right)\right\|_{L^{2}(\Omega)}
$$

for some $\kappa>0$ independent on $\delta$. Combining the above estimate with (3.2.10), we can find a constant $c>0$, independent of $\delta$, such that

$$
\|v\|_{L^{\infty}\left(\tau^{*}+\delta, T ; L^{2}(\Omega)\right)} \leq c \delta .
$$

Now, we define

$$
w(\cdot, t)= \begin{cases}z(\cdot, t) & \text { if } t \in\left[0, \tau^{*}+\delta\right] \\ \psi(\cdot, t) & \text { if } t \in\left(\tau^{*}+\delta, T\right]\end{cases}
$$

Clearly, $w(\cdot, 0)=0$ and $w(\cdot, T)=0$ in $\Omega$. Let $y^{*}$ be the solution of (3.2.1) with $(\tau, g)=$ $\left(\tau^{*}, g^{*}\right)$. Then $y^{*}(\cdot, T)=0$ in $\Omega$. Further, one can easily check that the function $y^{*}+w$ solves (3.2.7) with

$$
g(\cdot, t)= \begin{cases}0 & \text { if } t \in\left[0, \tau^{*}+\delta\right] \\ g^{*}(\cdot, t)+1_{\mid E} v(\cdot, t) & \text { if } t \in\left(\tau^{*}+\delta, T\right]\end{cases}
$$

Finally, we take $\delta \in\left(0, \delta_{0}\right)$ such that $c \delta \leq \varepsilon$. Then $g \in \mathcal{G}^{M}$. Indeed, it follows from $(3.2 .15),(3.2 .8)$ and (3.2.13) that

$$
\begin{aligned}
\|g(\cdot, t)\|_{L^{2}(\Omega)} & \leq\left\|g^{*}(\cdot, t)\right\|_{L^{2}(\Omega)}+\left\|1_{\mid E} v(\cdot, t)\right\|_{L^{2}(\Omega)} \\
& \leq \begin{cases}M-\varepsilon+c \delta & \text { a.e. if } t \in E \cap\left(\tau^{*}+\delta, T\right) \\
M & \text { a.e. if } t \notin E \cap\left(\tau^{*}+\delta, T\right) \\
0 & \text { if } t \in\left(0, \tau^{*}+\delta\right)\end{cases} \\
& \leq M \quad \text { for a.e. } t \in(0, T) .
\end{aligned}
$$

This completes the proof.

\section{Appendix}

Proof of Proposition 2.1

Since $|E|>0$, almost every point of $E$ is a point of density of $E$. Let $\ell \in(0, T)$ be such a point. Then

$$
\frac{\left|E^{c} \cap(\ell-\theta, \ell+\theta)\right|}{|(\ell-\theta, \ell+\theta)|} \rightarrow 0 \quad \text { and } \quad \frac{|E \cap(\ell-\theta, \ell+\theta)|}{|(\ell-\theta, \ell+\theta)|} \rightarrow 1 \quad \text { as } \theta \rightarrow 0 .
$$

Let $z>1$. Let $0<\epsilon \leq \min \left(\frac{z-1}{1+3 z}, \frac{1}{3}\right)$, which implies that

$$
\frac{\epsilon}{1-\epsilon} \frac{1+z}{z-1} \leq \frac{1}{2} \quad \text { and } \quad 1+\frac{\epsilon}{1-\epsilon} \leq \frac{3}{2}
$$


Then by (A.1.1), there exists $\theta_{0}=\theta_{0}(\epsilon)>0$ such that for any $\theta<\theta_{0}$,

$$
\frac{\left|E^{c} \cap(\ell-\theta, \ell+\theta)\right|}{|(\ell-\theta, \ell+\theta)|}<\epsilon \quad \text { and } \quad 1-\epsilon<\frac{|E \cap(\ell-\theta, \ell+\theta)|}{|(\ell-\theta, \ell+\theta)|}
$$

which implies that

$$
\left|E^{c} \cap(\ell-\theta, \ell+\theta)\right|<\frac{\epsilon}{1-\epsilon}|E \cap(\ell-\theta, \ell+\theta)| .
$$

Write $\widetilde{\theta}_{0}=\min \left(\theta_{0}, T-\ell\right)$. Let $\ell_{1}$ be such that $\ell<\ell_{1}<\ell+\tilde{\theta}_{0} \leq T$. Define $\left\{\ell_{m}\right\}_{m \geq 1}$ by (2.1.1). Clearly,

$$
\ell_{m}-\ell<\ell_{m-1}-\ell<\cdots<\ell_{1}-\ell<\tilde{\theta}_{0} \leq \theta_{0}
$$

and

$$
\ell_{m+1}-\ell_{m+2}=\frac{1}{z^{m+1}}(z-1)\left(\ell_{1}-\ell\right)
$$

Then

$$
\begin{aligned}
\ell_{m}-\ell_{m+1} & =\left|E^{c} \cap\left(\ell_{m+1}, \ell_{m}\right)\right|+\left|E \cap\left(\ell_{m+1}, \ell_{m}\right)\right| \\
& \leq\left|E^{c} \cap\left(2 \ell-\ell_{m}, \ell_{m}\right)\right|+\left|E \cap\left(\ell_{m+1}, \ell_{m}\right)\right| \\
& \leq \frac{\epsilon}{1-\epsilon}\left|E \cap\left(2 \ell-\ell_{m}, \ell_{m}\right)\right|+\left|E \cap\left(\ell_{m+1}, \ell_{m}\right)\right| .
\end{aligned}
$$

The first inequality in (A.1.6) follows from (2.1.1); in the second, we use (A.1.3) with $\theta=\ell_{m}-\ell$ and (A.1.4). Thus we have

$$
\begin{aligned}
\ell_{m}-\ell_{m+1} & \leq \frac{\epsilon}{1-\epsilon}\left[\left|E \cap\left(2 \ell-\ell_{m}, \ell_{m+1}\right)\right|+\left|E \cap\left(\ell_{m+1}, \ell_{m}\right)\right|\right]+\left|E \cap\left(\ell_{m+1}, \ell_{m}\right)\right| \\
& \leq\left(1+\frac{\epsilon}{1-\epsilon}\right)\left|E \cap\left(\ell_{m+1}, \ell_{m}\right)\right|+\frac{\epsilon}{1-\epsilon}\left[\ell_{m+1}-\left(2 \ell-\ell_{m}\right)\right] .
\end{aligned}
$$

Moreover, it follows from (2.1.1) and (A.1.5) that

$$
\ell_{m+1}-\left(2 \ell-\ell_{m}\right)=\frac{1}{z^{m}}(1+z)\left(\ell_{1}-\ell\right)=\frac{1+z}{z-1}\left(\ell_{m}-\ell_{m+1}\right) .
$$

This, along with (A.1.7) and (A.1.2), leads to (2.1.2).

\section{Some inequalities}

Suppose $a \in L^{\infty}\left(0, T ; L^{q}(\Omega)\right)$ where $q \geq 2$ for $n=1$, and $q>n$ for $n \geq 2$. Let

$$
p= \begin{cases}2 n / q & \text { if } n<q \leq 2 n \\ 1 & \text { if } 2 n \leq q\end{cases}
$$


Then, for each $\varepsilon>0$, there is $C_{(\varepsilon, \Omega, n, q)}>0$ such that for any $\phi \in H_{0}^{1}(\Omega)$ and for a.e. $t \in(0, L) \subset[0, T]$,

$$
\begin{aligned}
& \int_{\Omega}|a(x, t) \phi(x)|^{2} G_{\lambda}(x, t) d x \leq \varepsilon \int_{\Omega}|\nabla \phi(x)|^{2} G_{\lambda}(x, t) d x \\
& +C_{(\varepsilon, \Omega, n, q)}\left(\|a\|_{L^{\infty}\left(0, L ; L^{q}(\Omega)\right)}^{4 /(2-p)}+\frac{\|a\|_{L^{\infty}\left(0, L ; L^{q}(\Omega)\right)}^{2}}{(L-t+\lambda)^{p}}\right) \int_{\Omega}|\phi(x)|^{2} G_{\lambda}(x, t) d x
\end{aligned}
$$

and

$$
\begin{aligned}
& \int_{\Omega} a(x, t)|\phi(x)|^{2} G_{\lambda}(x, t) d x \leq \varepsilon \int_{\Omega}|\nabla \phi(x)|^{2} G_{\lambda}(x, t) d x \\
& +C_{(\varepsilon, \Omega, n, q)}\left(\|a\|_{L^{\infty}\left(0, L ; L^{q}(\Omega)\right)}^{2}+\frac{\|a\|_{L^{\infty}\left(0, L ; L^{q}(\Omega)\right)}}{L-t+\lambda}\right) \int_{\Omega}|\phi(x)|^{2} G_{\lambda}(x, t) d x .
\end{aligned}
$$

Proof of (A.2.1) and (A.2.2). Notice that $1 \leq p<2$. If $n \geq 2$, then

$$
\begin{aligned}
& \int_{\Omega}|a \phi|^{2} G_{\lambda} d x \leq\left\|a^{2}\right\|_{L^{n / p}(\Omega)}\left\|\phi^{2} G_{\lambda}\right\|_{L^{n /(n-p)(\Omega)}} \quad \text { (by Hölder inequality) } \\
& \leq\|a\|_{L^{2 n / p}(\Omega)}^{2}\left\|\left(\phi^{2} G_{\lambda}\right)^{1 / p}\right\|_{L^{p n /(n-p)}(\Omega)}^{p} \\
& \leq C_{(\Omega, n, q)}\|a\|_{L^{q}(\Omega)}^{2}\left\|\nabla\left(\left(\phi^{2} G_{\lambda}\right)^{1 / p}\right)\right\|_{L^{p}(\Omega)}^{p} \quad \text { (by Sobolev inequality) } \\
& \leq C_{(\Omega, n, q)}\|a\|_{L^{q}(\Omega)}^{2} \int_{\Omega}\left(|\phi|^{2-p}|\nabla \phi|^{p} G_{\lambda}+|\phi|^{2}\left(G_{\lambda}\right)^{1-p}\left|\nabla G_{\lambda}\right|^{p}\right) d x \\
& \leq C_{(\Omega, n, q)}\|a\|_{L^{q}(\Omega)}^{2}\left(\int_{\Omega}|\phi|^{2} G_{\lambda} d x\right)^{(2-p) / 2}\left(\int_{\Omega}|\nabla \phi|^{2} G_{\lambda} d x\right)^{p / 2} \text { (by Hölder inequality) } \\
& +C_{(\Omega, n, q)}\|a\|_{L^{q}(\Omega)}^{2}\left(\frac{1}{|L-t+\lambda|^{p}} \int_{\Omega}|\phi|^{2} G_{\lambda} d x\right) \\
& \leq C_{(\Omega, n, q)}\|a\|_{L^{q}(\Omega)}^{2}\left(\varepsilon \int_{\Omega}|\nabla \phi|^{2} G_{\lambda} d x+\left(\frac{1}{\varepsilon^{p /(2-p)}}+\frac{1}{|L-t+\lambda|^{p}}\right) \int_{\Omega}|\phi|^{2} G_{\lambda} d x\right),
\end{aligned}
$$

and

$$
\begin{aligned}
\int_{\Omega} a|\phi|^{2} G_{\lambda} d x \leq\|a\|_{L^{n}(\Omega)}\left\|\phi^{2} G_{\lambda}\right\|_{L^{n /(n-1)}(\Omega)} \quad \text { (by Hölder inequality) } & \\
& \leq C_{(\Omega, n)}\|a\|_{L^{n}(\Omega)}\left\|\nabla\left(\phi^{2} G_{\lambda}\right)\right\|_{L^{1}(\Omega)} \quad \text { (by Sobolev inequality) } \\
& \leq C_{(\Omega, n, q)}\|a\|_{L^{q}(\Omega)} \int_{\Omega}\left(|\phi||\nabla \phi| G_{\lambda}+|\phi|^{2}\left|\nabla G_{\lambda}\right|\right) d x \quad \text { (by Hölder inequality) } \\
& \leq C_{(\Omega, n, q)}\|a\|_{L^{q}(\Omega)}\left(\varepsilon \int_{\Omega}|\nabla \phi|^{2} G_{\lambda} d x+\left(\frac{1}{\varepsilon}+\frac{1}{|L-t+\lambda|}\right) \int_{\Omega}|\phi|^{2} G_{\lambda} d x\right) .
\end{aligned}
$$


If $n=1$, then

$$
\begin{aligned}
& \int_{\Omega}|a \phi|^{2} G_{\lambda} d x \leq\left\|a^{2}\right\|_{L^{1}(\Omega)}\left\|\phi^{2} G_{\lambda}\right\|_{L^{\infty}(\Omega)} \quad \text { (by Hölder inequality) } \\
& \leq C_{(\Omega)}\|a\|_{L^{2}(\Omega)}^{2}\left\|\nabla\left(\phi^{2} G_{\lambda}\right)\right\|_{L^{1}(\Omega)} \quad \text { (by Sobolev inequality) } \\
& \leq C_{(\Omega)}\|a\|_{L^{2}(\Omega)}^{2} \int_{\Omega}\left(|\phi||\nabla \phi| G_{\lambda}+|\phi|^{2}\left|\nabla G_{\lambda}\right|\right) d x \\
& \leq C_{(\Omega)}\|a\|_{L^{2}(\Omega)}^{2}\left(\int_{\Omega}|\phi|^{2} G_{\lambda} d x\right)^{1 / 2}\left(\int_{\Omega}|\nabla \phi|^{2} G_{\lambda} d x\right)^{1 / 2} \quad \text { (by Hölder inequality) } \\
& +C_{(\Omega)}\|a\|_{L^{2}(\Omega)}^{2}\left(\frac{1}{|L-t+\lambda|} \int_{\Omega}|\phi|^{2} G_{\lambda} d x\right) \\
& \leq C_{(\Omega)}\|a\|_{L^{2}(\Omega)}^{2}\left(\varepsilon \int_{\Omega}|\nabla \phi|^{2} G_{\lambda} d x+\left(\frac{1}{\varepsilon}+\frac{1}{|L-t+\lambda|}\right) \int_{\Omega}|\phi|^{2} G_{\lambda} d x\right),
\end{aligned}
$$

and

$$
\begin{aligned}
& \int_{\Omega} a|\phi|^{2} G_{\lambda} d x \leq\|a\|_{L^{1}(\Omega)}\left\|\phi^{2} G_{\lambda}\right\|_{L^{\infty}(\Omega)} \quad \text { (by Hölder inequality) } \\
& \quad \leq C_{(\Omega)}\|a\|_{L^{1}(\Omega)}\left\|\nabla\left(\phi^{2} G_{\lambda}\right)\right\|_{L^{1}(\Omega)} \quad \text { (by Sobolev inequality) } \\
& \quad \leq C_{(\Omega)}\|a\|_{L^{1}(\Omega)} \int_{\Omega}\left(|\phi||\nabla \phi| G_{\lambda}+|\phi|^{2}\left|\nabla G_{\lambda}\right|\right) d x \quad \text { (by Hölder inequality) } \\
& \quad \leq C_{(\Omega)}\|a\|_{L^{2}(\Omega)}\left(\varepsilon \int_{\Omega}|\nabla \phi|^{2} G_{\lambda} d x+\left(\frac{1}{\varepsilon}+\frac{1}{|L-t+\lambda|}\right) \int_{\Omega}|\phi|^{2} G_{\lambda} d x\right) \quad \forall \varepsilon>0 .
\end{aligned}
$$

Acknowledgments. This work was partially supported by the National Basis Research Program of China (973 Program) under grant 2011CB808002 and the National Science Foundation of China under grants 11161130003 and 11171264.

\section{References}

[BT] Bardos, C., Tartar, L.: Sur l'unicité retrograde des équations paraboliques et quelques questions voisines. Arch. Ration. Mech. Anal. 50, 10-25 (1973) Zbl 0258.35039 MR 0338517

[DFGZ] Doubova, A., Fernández-Cara, E., González-Burgos, M., Zuazua, E.: On the controllability of parabolic systems with a nonlinear term involving the state and the gradient. SIAM J. Control Optim. 41, 798-819 (2002) Zbl 1038.93041 MR 1939871

[DZZ] Duyckaerts, T., Zhang, X., Zuazua, E.: On the optimality of the observability inequalities for parabolic and hyperbolic systems with potentials. Ann. Inst. H. Poincaré Anal. Non Linéaire 25, 1-41 (2008) Zbl 1248.93031 MR 2383077

[EFV] Escauriaza, L., Fernández, F. J., Vessella, S.: Doubling properties of caloric functions. Appl. Anal. 85, 205-223 (2006) Zbl 1090.35050 MR 2198840

[FPZ] Fabre, C., Puel, J.-P., Zuazua, E.: Contrôlabilité approchée de l'équation de la chaleur linéaire avec des contrôles de norme $L^{\infty}$ minimal. C. R. Acad. Sci. Paris Sér. I Math. 316, 679-684 (1993) Zbl 0799.35094 MR 1214415 
[F] Fattorini, H.: Infinite Dimensional Linear Control Systems; the Time Optimal and Norm Optimal Problems. North-Holland Math. Stud. 201, Elsevier, Amsterdam (2005) Zbl 1135.93001 MR 2158806

[FI] Fursikov, A. V., Imanuvilov, O. Y.: Controllability of Evolution Equations. Lecture Notes Ser. 34, Seoul National Univ., Seoul (1996) Zbl 0862.49004 MR 1406566

[K] Kenig, C.: Quantitative unique continuation, logarithmic convexity of gaussian means and Hardy's uncertainty principle. In: Proc. Sympos. Pure Math. 79, Volume in celebration of V. Mazya's 70 birthday, 207-227 (2008) Zbl 1155.35450 MR 2500494

[KT] Koch, H., Tataru, D.: Carleman estimates and unique continuation for second order parabolic equations with nonsmooth coefficients. Comm. Partial Differential Equations 34, 305-366 (2009) Zbl 1178.35107 MR 2530700

[LR] Lebeau, G., Robbiano, L.: Contrôle exacte de l'équation de la chaleur. Comm. Partial Differential Equations 20, 335-356 (1995) Zbl 0819.35071 MR 1312710

[L] Lin, F. H.: Remarks on a backward parabolic problem. Methods Appl. Anal. 10, 245-252 (2003) Zbl 1085.35026 MR 2074750

[Li] Lions, J.-L.: Optimal Control of Systems Governed by Partial Differential Equations. Springer (1971) Zbl 0203.09001 MR 0271512

[M] Miller, L.: A direct Lebeau-Robbiano strategy for the observability of heat-like semigroups. Discrete Contin. Dynam. Systems Ser. B 14, 1465-1485 (2010) Zbl 1219.93017 MR 2679651

[MS] Mizel, V., Seidman, T.: An abstract 'bang-bang principle' and time optimal boundary control of the heat equation. SIAM J. Control Optim. 35, 1204-1216(1997) Zbl 0891.49014 MR 1453296

[PW] Phung, K. D., Wang, G.: Quantitative unique continuation for the semilinear heat equation in a convex domain. J. Funct. Anal. 259, 1230-1247 (2010) Zbl 1215.35042 MR 2652187

[P] Poon, C. C.: Unique continuation for parabolic equations. Comm. Partial Differential Equations 21, 521-539 (1996) Zbl 0852.35055 MR 1387458

[W] Wang, G.: $L^{\infty}$-null controllability for the heat equation and its consequences for the time optimal control problem. SIAM J. Control Optim. 47, 1701-1720 (2008) Zbl 1165.93016 MR 2421326

[Z] Zuazua, E.: Controllability and observability of partial differential equations: Some results and open problems. In: Handbook of Differential Equations: Evolutionary Differential Equations, Vol. 3, Elsevier, 527-621 (2006) Zbl 1193.35234 MR 2549374 